\title{
On the Perturbation Theory of Self-Adjoint Operators
}

\author{
Sergio ALBEVERIO, ${ }^{\mathrm{a}}$ Sergei KUZHEL ${ }^{\mathrm{b}}$ and Leonid P. NIZHNIK ${ }^{\mathrm{b}}$ \\ ${ }^{\mathrm{a}}$ Universität Bonn and ${ }^{\mathrm{b}}$ National Academy of Sciences of Ukraine \\ (Communicated by T. Kawasaki)
}

\begin{abstract}
We show that all types of self-adjoint perturbations of a semi-bounded operator $A$ (purely singular, mixed singular, and regular) can be described and studied from a unique point of view in the framework of the extension theory as well as in the framework of the additive perturbation theory. We also show that any singular finite rank perturbation $\widetilde{A}$ can be approximated in the norm resolvent sense by regular finite rank perturbations of $A$. An application is given to the study of Schrödinger operators with point interactions.
\end{abstract}

\section{Introduction}

Let $A$ be a semi-bounded self-adjoint operator acting in a separable Hilbert space $\mathcal{H}$ with inner product $(\cdot, \cdot)$ and let $\mathcal{D}(A), \mathcal{R}(A)$, $\operatorname{ker} A$, and $\rho(A)$ denote the domain, the range, the null space and the resolvent set of $A$, respectively. Without loss of generality, we will assume that $A \geq I$. By $P_{M}$ we denote an orthoprojector in $\mathcal{H}$ onto a closed subspace $M$ of $\mathcal{H}$.

Let $N$ be a closed subspace of $\mathcal{H}$. Define $\mathcal{D}\left(A_{N}\right)$ and $A_{N}$ as

$$
\mathcal{D}\left(A_{N}\right)=\{u \in \mathcal{D}(A) \mid(A u, \eta)=0, \forall \eta \in N\}, \quad A_{N}=A\left\lceil\mathcal{D}\left(A_{N}\right),\right.
$$

where $A\left\lceil\mathcal{D}\left(A_{N}\right)\right.$ means the restriction of $A$ onto $\mathcal{D}\left(A_{N}\right)$. Obviously, $A_{N}$ is a closed symmetric operator in $\mathcal{H}$ and its defect subspace $\mathcal{R}\left(A_{N}\right)^{\perp}=\mathcal{H} \ominus \mathcal{R}\left(A_{N}\right)$ coincides with $N$. The operator $A_{N}$ is densely defined if and only if $N \cap \mathcal{D}(A)=\{0\}$.

Let $\widetilde{A}$ be a self-adjoint operator in $\mathcal{H}$ and let $\widetilde{A} \wedge A$ be the maximal common part of $\widetilde{A}$ and $A$ :

$$
\widetilde{A} \wedge A=A \uparrow_{\mathcal{D}(\tilde{A} \wedge A)}, \quad \mathcal{D}(\widetilde{A} \wedge A)=\{u \in \mathcal{D}(A) \cap \mathcal{D}(\widetilde{A}) \mid A u=\widetilde{A} u\} .
$$

Comparing (1.1), (1.2) one gets $\widetilde{A} \wedge A=A_{N}$, where

$$
N=\{\eta \in \mathcal{H} \mid(A u, \eta)=0, A u=\widetilde{A} u\}
$$

is the defect subspace of $\widetilde{A} \wedge A$. The operators $\widetilde{A}$ and $A$ are relatively prime extensions of the symmetric operator $A_{N}$ (i.e., the maximal common part $\widetilde{A} \wedge A$ of $\widetilde{A}$ and $A$ coincides

Received October 23, 2006

1991 Mathematics Subject Classification: 47A10, 47A55, 81Q10

Key words: self-adjoint perturbations, boundary value spaces, Krein's resolvent formula, additive perturbations, finite rank perturbations, resolvent approximation, Schrödinger operator, point interactions 
with $A_{N}$ ) and the self-adjoint extension $\widetilde{A}$ can be considered as a perturbation of the initial (nonperturbed) operator $A$. In this way, an arbitrary self-adjoint operator $\widetilde{A} \neq A$ can be regarded as a perturbation of a fixed operator $A$.

The perturbation $\widetilde{A}$ is finite rank if and only if $\operatorname{dim} N<\infty$, where $N$ is the defect subspace of $\widetilde{A} \wedge A$.

A self-adjoint operator $\widetilde{A} \neq A$ is called: regular perturbation of $A$ if $\mathcal{D}(A) \cap \mathcal{D}(\widetilde{A})=$ $\mathcal{D}(A)$ and singular perturbation if $\mathcal{D}(A) \cap \mathcal{D}(\widetilde{A}) \neq \mathcal{D}(A)$. It is convenient to divide the class of singular perturbations into two subclasses. Precisely, we will say that a singular perturbation $\widetilde{A}$ is purely singular if the maximal common part $\widetilde{A} \wedge A$ is densely defined and mixed singular if $\widetilde{A} \wedge A$ is nondensely defined.

At present, regular and purely singular perturbations are well-studied, respectively, by the additive perturbation theory and extension theory methods (see $[1,2,16]$ and the reference list therein). Mixed singular perturbations are investigated considerably less.

In the present paper, a unique approach to the description and study of all types of perturbations of $A$ (regular, purely singular, and mixed singular) is developed.

In Section 2, we describe all self-adjoint operators $\widetilde{A}$ in relation to a fixed self-adjoint operator $A$ by parameters $\{N, T\}$. Here $N$ is a defect subspace of $\widetilde{A} \wedge A$ and a self-adjoint (in $N$ ) operator $T$ distinguishes $\widetilde{A}$ among self-adjoint extensions of $\widetilde{A} \wedge A$ which are relatively prime to $A$. We study the relationship $\widetilde{A} \leftrightarrow\{N, T\}$ in detail and establish simple connections between $\{N, T\}$ and the parameters characterizing $\widetilde{A}$ in the frameworks of the extension and additive perturbation theories. (The relationship between such approaches for finite rank perturbations has been studied in $[25,27]$.)

Our investigations show that the parameters $\{N, T\}$ are quite natural for the description of self-adjoint perturbations of a semi-bounded operator $A$. In particular, they naturally appear in the Krein's resolvent formula relating $\widetilde{A}$ and $A$ (Theorem 2.1). The parametrization $\widetilde{A} \leftrightarrow$ $\{N, T\}$ provides convenient tools for extracting different properties of $\widetilde{A}$. For example, the Krein-von Neumann extension of $A_{N}$ corresponds to the pair $\{N, 0\}$ (Proposition 2.2) and the Friedrichs extension is determined by $\{N, T\}$, where $N \subset \mathcal{D}\left(A^{1 / 2}\right), N \cap \mathcal{D}(A)=\{0\}$ and $T$ is uniquely defined by the relation $(T \eta, \xi)=\left(A^{1 / 2} \eta, A^{1 / 2} \xi\right), \forall \eta, \xi \in N$ (Proposition 3.3).

In a certain sense, some results of Section 2 (Theorem 2.2) were inspired by the recent papers $[21,22]$ where the authors present various ways of description of all self-adjoint perturbations of $A$ (and moreover, all closed operators having at least one common point in their resolvent sets) by parameters $\{M, \gamma\}$ appearing in Krein's type resolvent formulas. In Remark 1 , we present an explicit formula relating the parameters $\{M, \gamma\}$ and $\{N, T\}$.

By the construction, the parameters $\{N, T\}$ deal with the extension theory approach. In Theorems 2.5, 2.6, we reformulate the description of self-adjoint perturbations in the framework of the additive perturbation theory. To do this, the parameters $\{N, B, R\}$ are used. Here, $B$ and $R$ are self-adjoint in $N$ such that $B=(T-R)^{-1}$.

In Section 3, self-adjoint perturbations $\widetilde{A}$ of $A$ are described as $A+V$, where, in general, the "potentials" $V$ act in the scale of Hilbert spaces associated with $A$ ( $A$-scale). 
In Section 4, we show that any finite rank self-adjoint perturbation $\widetilde{A}$ can be approximated by regular perturbations in the norm resolvent sense. Section 5 deals with some application for the Schrödinger operators with point interactions.

\section{Extension theory approach}

In this section we are going to describe all types of perturbations of $A$ (purely singular, mixed singular, and regular) by methods of the extension theory.

2.1. Preliminary results. The boundary value spaces (BVS) approach is one of the most popular methods for the description of self-adjoint extensions of a given densely defined symmetric operator $[13,23]$. Now we briefly outline some well-known results of the BVStheory needed for our exposition.

Definition 2.1 ([13]). Let $L_{\text {min }}$ be a closed densely defined symmetric operator in $\mathcal{H}$. A triple $\left(\mathfrak{N}, \Gamma_{0}, \Gamma_{1}\right)$, where $\mathfrak{N}$ is an auxiliary Hilbert space and $\Gamma_{0}, \Gamma_{1}$ are linear mappings of $\mathcal{D}\left(L_{\min }^{*}\right)$ into $\mathfrak{N}$, is called a boundary value space (BVS) of $L_{\min }^{*}$ if the abstract Green identity

$$
\left(L_{\text {min }}^{*} f, g\right)-\left(f, L_{\min }^{*} g\right)=\left(\Gamma_{1} f, \Gamma_{0} g\right)_{\mathfrak{N}}-\left(\Gamma_{0} f, \Gamma_{1} g\right)_{\mathfrak{N}}, \quad f, g \in \mathcal{D}\left(L_{\text {min }}^{*}\right)
$$

is satisfied and the map $\left(\Gamma_{0}, \Gamma_{1}\right): \mathcal{D}\left(L_{\text {min }}^{*}\right) \rightarrow \mathfrak{N} \oplus \mathfrak{N}$ is surjective.

If ( $\left.\mathfrak{N}, \Gamma_{0}, \Gamma_{1}\right)$ is a BVS of $L_{\min }^{*}$, then the restrictions of the adjoint operator $L_{\min }^{*}$ onto the domains $\operatorname{ker} \Gamma_{0}$ and $\operatorname{ker} \Gamma_{1}$ determine, respectively, self-adjoint extensions $L_{0}$ and $L_{1}$ of $L_{\min }$.

The operators $L_{0}$ and $L_{1}$ are transversal extensions of $L_{\min }$, i.e., they are relatively prime with respect to $L_{\text {min }}: L_{0} \wedge L_{1}=L_{\text {min }}$ and $\mathcal{D}\left(L_{0}\right)+\mathcal{D}\left(L_{1}\right)=\mathcal{D}\left(L_{\text {min }}^{*}\right)$. These properties are characteristic for the existence of BVS. Precisely, for any transversal self-adjoint extensions $L_{0}$ and $L_{1}$ of $L_{\min }$ there exists a BVS $\left(\mathfrak{N}, \Gamma_{0}, \Gamma_{1}\right)$ of $L_{\min }^{*}$ such that $L_{0}=L_{\min }^{*} \Upsilon_{\operatorname{ker} \Gamma_{0}}$ and $L_{1}=L_{\min }^{*} \Upsilon_{\operatorname{ker} \Gamma_{1}}$.

Lemma 2.1 ([11,13]). Let $L$ be a fixed self-adjoint extension of $L_{\min }$ and let $\left(\mathfrak{N}, \Gamma_{0}, \Gamma_{1}\right)$ be a BVS of $L_{\min }^{*}$ such that $\mathcal{D}(L)=\operatorname{ker} \Gamma_{1}$. Then an arbitrary self-adjoint extension $\widetilde{L}$ of $L_{\min }$ relatively prime with $L$ is defined by the formula

$$
\widetilde{L}=L_{\min }^{*}\left\lceil_{\mathcal{D}(\widetilde{L})}, \quad \mathcal{D}(\widetilde{L})=\left\{f \in \mathcal{D}\left(L_{\min }^{*}\right) \mid T \Gamma_{1} f=\Gamma_{0} f\right\},\right.
$$

where $T$ is a self-adjoint operator in $\mathfrak{N}$. The correspondence $\widetilde{L} \leftrightarrow T$ determined by (2.1) is a bijection between the set of all self-adjoint extensions $\widetilde{L}$ relatively prime with $L$ and the set of all self-adjoint operators in $\mathfrak{N}$. Self-adjoint extensions $\widetilde{L}$ transversal with $L$ are determined by bounded self-adjoint operators $T$.

Let us use Lemma 2.1 for the description of purely singular perturbations $\widetilde{A}$ of $A$. In that case $\widetilde{A}$ and $A$ are relatively prime self-adjoint extensions of the densely defined symmetric operator $A_{N}=\widetilde{A} \wedge A$, where the defect subspace $N$ of $\widetilde{A} \wedge A$ satisfies the relation $N \cap \mathcal{D}(A)=$ 
$\{0\}$. The domain of the adjoint operator $A_{N}^{*}$ has the form $\mathcal{D}\left(A_{N}^{*}\right)=\mathcal{D}(A) \dot{+} N$ and

$$
A_{N}^{*} f=A_{N}^{*}(u+\eta)=A u, \quad \forall f=u+\eta \in \mathcal{D}\left(A_{N}^{*}\right)(u \in \mathcal{D}(A), \eta \in N) .
$$

Taking into account (see e.g., [13]) that the $\operatorname{triple}^{1}\left(N, \Gamma_{0}, \Gamma_{1}\right)$, where

$$
\Gamma_{0}(u+\eta)=P_{N} A u, \quad \Gamma_{1}(u+\eta)=-\eta \quad(\forall u \in \mathcal{D}(A), \quad \forall \eta \in N)
$$

is a BVS of $A_{N}^{*}$ such that $\operatorname{ker} \Gamma_{1}=\mathcal{D}(A)$ and applying Lemma 2.1 (for $\mathfrak{N}=N, L=$ $A, L_{\min }=A_{N}$ ) with the use of (2.2) and (2.3), we immediately obtain

PROPOSITION 2.1. A purely singular perturbation $\widetilde{A}$ of $A$ is determined by the formula

$\widetilde{A} f=\widetilde{A}(u+\eta)=A u, \quad \mathcal{D}(\widetilde{A})=\left\{f=u+\eta, u \in \mathcal{D}(A), \eta \in \mathcal{D}(T) \mid-T \eta=P_{N} A u\right\}$, where $N$ is the defect subspace of $\widetilde{A} \wedge A$ and $T$ is a self-adjoint operator in $N$.

The correspondence $\widetilde{A} \leftrightarrow\{N, T\}$ is a bijection between the set of all purely singular perturbations of $A$ and the set of pairs $\{N, T\}$, where $N$ is an arbitrary non-trivial subspace of $\mathcal{H}$ such that $N \cap \mathcal{D}(A)=\{0\}$ and $T$ is an arbitrary self-adjoint operator in $N$.

In the case of mixed singular or regular perturbations $\widetilde{A}$, the situation is more complicated because the operator $A_{N}=\widetilde{A} \wedge A$ is non-densely defined and the adjoint $A_{N}^{*}$ does not exist as a uniquely defined operator. As a rule (see e.g., $[9,10,19]), A_{N}$ and its 'adjoint' are understood as linear relations and the description of all self-adjoint relations that are extensions of the graph of $A_{N}$ is carried out.

The approaches presented below enable one to preserve the operator form of the exposition and they do not need the linear relations technique.

2.2. The description of all self-adjoint perturbations of $A$. Let $\widetilde{A} \neq A$ be an arbitrary self-adjoint operator in $\mathcal{H}$. Obviously, $\widetilde{A}$ and $A$ are relatively prime with respect to $A_{N}=\widetilde{A} \wedge A$ but the symmetric operator $A_{N}$ is not necessarily densely defined and its defect subspace $N$ may have a non-trivial intersection with $\mathcal{D}(A)$.

Now, we are going to extend the description of purely singular perturbations presented in Proposition 2.1 to the general case of all self-adjoint perturbations.

LEMMA 2.2. Assume that $A$ is an invertible operator in $\mathcal{H}, N$ is a subspace of $\mathcal{H}$, and $T$ is a linear operator in $N$. Then the relations

$$
\begin{gathered}
A_{N, T} f=A_{N, T}(u+\eta)=A u, \\
\mathcal{D}\left(A_{N, T}\right)=\left\{f=u+\eta, u \in \mathcal{D}(A), \eta \in \mathcal{D}(T) \mid-T \eta=P_{N} A u\right\}
\end{gathered}
$$

define an operator $A_{N, T}$ in $\mathcal{H}$ if and only if $T$ satisfies the condition

$$
T \eta-P_{N} A \eta=0 \Rightarrow \eta=0, \quad \forall \eta \in \mathcal{D}(A) \cap \mathcal{D}(T) .
$$

\footnotetext{
${ }^{1}$ In fact, this BVS was already implicitly used in the classical works $[8,20]$.
} 
Proof. Assume that $f=u+\eta \in \mathcal{D}\left(A_{N, T}\right)$ and $f=0$. Then $\eta=-u \in$ $\mathcal{D}(A) \cap \mathcal{D}(T)$. In this case, (2.4) and (2.5) give $A_{N, T} f=-A \eta$ and $T \eta=P_{N} A \eta$. Since $A$ is invertible, the implication $f=0 \Rightarrow A_{N, T} f=0$ holds if and only if $\eta=0$ that is equivalent to (2.6).

Definition 2.2. An operator $T$ acting in a subspace $N$ of $\mathcal{H}$ is said to be admissible with respect to $A$ if $T$ satisfies (2.6).

It follows from (1.1), (2.4), and (2.5) that $A_{N, T}$ is an extension of $A_{N}$ for an arbitrary admissible operator $T$.

THEOREM 2.1. Assume that $A \geq I$ is a self-adjoint operator in $\mathcal{H}, N$ is a subspace of $\mathcal{H}$, and $T$ is an admissible operator in $N$. Then the operator $A_{N, T}$ determined by (2.4), (2.5) is a self-adjoint extension of the symmetric operator $A_{N}$ if and only if $T$ is self-adjoint. In that case $z \in \rho(A) \cap \rho\left(A_{N, T}\right)$ if and only if $Q_{0}(z)+T$ is boundedly invertible, where $Q_{0}(z)=z P_{N} A(A-z I)^{-1} P_{N}$ and

$$
\left(A_{N, T}-z I\right)^{-1}=(A-z I)^{-1}-A(A-z I)^{-1}\left(Q_{0}(z)+T\right)^{-1} P_{N} A(A-z I)^{-1} .
$$

Proof. The domain $\mathcal{D}\left(A_{N, T}\right)$ determined by (2.5) can be also described as

$$
\mathcal{D}\left(A_{N, T}\right)=\mathcal{D}\left(A_{N}\right) \dot{+}\left\{\left(I-A^{-1} T\right) \eta \mid \forall \eta \in \mathcal{D}(T)\right\} .
$$

So, any $f \in \mathcal{D}\left(A_{N, T}\right)$ admits the representation $f=u_{0}-A^{-1} T \eta+\eta \quad\left(\forall u_{0} \in \mathcal{D}\left(A_{N}\right)\right.$, $\forall \eta \in \mathcal{D}(T)$ ) and the formula (2.4) takes the form

$$
A_{N, T} f=A_{N, T}\left(u_{0}-A^{-1} T \eta+\eta\right)=A u_{0}-T \eta .
$$

The formulas (2.4), (2.5) and (2.8), (2.9) give equivalent descriptions of $A_{N, T}$.

In view of (2.8), (2.9), $A_{N, T}$ is an extension of the symmetric operator $A_{N}$ for any choice of admissible operator $T$ in $N$.

Assume that $T$ is self-adjoint in $N$ and show that $A_{N, T}$ is densely defined in $\mathcal{H}$. Let $t \in \mathcal{H}$ be orthogonal to $\mathcal{D}\left(A_{N, T}\right)$. By virtue of (2.8), $t$ is orthogonal to $\mathcal{D}\left(A_{N}\right)$. This means that $t=A w_{r}$, where $w_{r} \in N_{r}=N \cap \mathcal{D}(A)$. Recalling that $t$ has to be orthogonal to all terms in (2.8), we get

$$
0=\left(-A^{-1} T \eta+\eta, A w_{r}\right)=\left(\eta, A w_{r}\right)-\left(T \eta, w_{r}\right), \quad \forall \eta \in \mathcal{D}(T) .
$$

Since $T$ is a self-adjoint operator in $N$, the obtained equality implies $w_{r} \in \mathcal{D}(T)$ and $T w_{r}=$ $P_{N} A w_{r}$. The last relation and (2.6) give $w_{r}=0$. Hence $t=0$ and $A_{N, T}$ is a densely defined operator in $\mathcal{H}$.

To find the adjoint $A_{N, T}^{*}$ we describe all elements $g, w \in \mathcal{H}$ satisfying the relation

$$
\left(A_{N, T} f, g\right)=(f, w), \quad \forall f \in \mathcal{D}\left(A_{N, T}\right) .
$$

Putting $f=u_{0} \in \mathcal{D}\left(A_{N}\right)$ in (2.10) and taking into account that $\left(u_{0}, w\right)=\left(A u_{0}, A^{-1} w\right)$, we get $g-A^{-1} w \in N$. Denote $\widetilde{\eta}=g-A^{-1} w$ and substitute $g=A^{-1} w+\widetilde{\eta}$ and elements 
$f \in \mathcal{D}\left(A_{N, T}\right)$ of the form $f=-A^{-1} T \eta+\eta \in \mathcal{D}\left(A_{N, T}\right)$ into (2.10). As a result, after a trivial transformations with the use of (2.9), we derive

$$
(-T \eta, \tilde{\eta})=(\eta, w), \quad \forall \eta \in \mathcal{D}(T) .
$$

Since $T$ is self-adjoint, (2.11) implies $\widetilde{\eta} \in \mathcal{D}(T)$ and $-T \widetilde{\eta}=P_{N} w$. But then, the element $g$ in (2.10) takes the form

$$
g=A^{-1}\left(I-P_{N}\right) w+A^{-1} P_{N} w+\widetilde{\eta}=u_{0}-A^{-1} T \widetilde{\eta}+\widetilde{\eta} \quad\left(u_{0}=A^{-1}\left(I-P_{N}\right) w\right) .
$$

Therefore, $g \in \mathcal{D}\left(A_{N, T}\right)$ and $A_{N, T} g=w$ (by (2.8) and (2.9)). So, if $T$ is self-adjoint, then $A_{N, T}^{*}=A_{N, T}$.

Let $A_{N, T}$ be self-adjoint. It follows from (2.4) and (2.5) that

$$
0=\left(A_{N, T} f, g\right)-\left(f, A_{N, T} g\right)=-(T \eta, \widetilde{\eta})+(\eta, T \widetilde{\eta})
$$

for an arbitrary $f=u+\eta$ and $g=v+\widetilde{\eta}$ from $\mathcal{D}\left(A_{N, T}\right)$. Hence, $T$ is a symmetric operator. To prove its self-adjointness, we consider an arbitrary $\widetilde{\eta} \in N$ and $w \in \mathcal{H}$ satisfying (2.11). In that case, as follows from above, $g=A^{-1} w+\widetilde{\eta}$ satisfies (2.10) for all $f \in \mathcal{D}\left(A_{N, T}\right)$. Hence $g \in \mathcal{D}\left(A_{N, T}\right)$ and $w=A_{N, T} g$ (since $A_{N, T}$ is self-adjoint). Combining this with (2.4), (2.5) one gets $\widetilde{\eta} \in \mathcal{D}(T)$ and $-T \widetilde{\eta}=P_{N} A u=P_{N} w$ (here $u=A^{-1} w$ ). Recalling now (2.11), we derive the self-adjointness of $T$.

To prove (2.7), we fix $z \in \mathbb{C} \backslash \mathbb{R}$ and consider the operator $Q_{0}(z)=z P_{N} A(A-z I)^{-1} P_{N}$. Since its imaginary part $\operatorname{Im} Q(z)=(\operatorname{Im} z) P_{N} A(A-\bar{z} I)^{-1} A(A-z I)^{-1} P_{N}$ is a positive (for $\operatorname{Im} z>0$ ) or negative (Im $z<0$ ) defined bounded operator in $N$ and $T$ is self-adjoint, the operator $Q_{0}(z)+T$ is boundedly invertible (i.e., $0 \in \rho\left(Q_{0}(z)+T\right)$ ).

Let $h \in \mathcal{H}$ and $f=u+\eta \in \mathcal{D}\left(A_{N, T}\right)$ be such that $h=\left(A_{N, T}-z I\right) f$. Using (2.4), (2.5), one gets $h=(A-z I) u-z \eta$ or $P_{N} A(A-z I)^{-1} h=-\left(Q_{0}(z)+T\right) \eta$ and hence, $\eta=-\left(Q_{0}(z)+T\right)^{-1} P_{N} A(A-z I)^{-1} h$.

On the other hand, it is easy to verify that $\left[\left(A_{N, T}-z I\right)^{-1}-(A-z I)^{-1}\right] h=$ $A(A-z I)^{-1} \eta$. Comparing the latter two equalities we justify (2.7) for $\operatorname{Im} z \neq 0$. Obviously, (2.7) can be extended onto $z \in \rho(A) \cap \rho\left(A_{N, T}\right)$ by continuity and $0 \in \rho\left(Q_{0}(z)+T\right) \Leftrightarrow$ $z \in \rho(A) \cap \rho\left(A_{N, T}\right)$. Theorem 2.1 is proved.

THEOREM 2.2. Let $\widetilde{A}$ be an arbitrary self-adjoint operator in $\mathcal{H}$ and $A \geq I$. Then there exists a unique pair $\{N, T\}$, where $T$ is an admissible self-adjoint operator in a subspace $N$ such that $\tilde{A}=A_{N, T}$.

The correspondence $\widetilde{A} \leftrightarrow\{N, T\}$ is a bijection between all self-adjoint operators and the set of pairs $\{N, T\}$, where $N$ is an arbitrary subspace of $\mathcal{H}$ and $T$ is an arbitrary admissible self-adjoint operator in $N$.

Proof. Assume that $\widetilde{A} \neq A$ is an arbitrary self-adjoint operator in $\mathcal{H}$. Then $\widetilde{A}$ and $A$ are relatively prime extensions of the symmetric (not necessarily densely defined) operator $A_{N}=\widetilde{A} \wedge A$, where $N$ is determined by (1.3). 
Denote by $N_{z}=\mathcal{H} \ominus \mathcal{R}\left(A_{N}-\bar{z} I\right)$ the defect subspace of $A_{N}$ corresponding to $z \in \mathbb{C}$. An analog of the von-Neumann formulas for non-densely defined symmetric operators (see e.g., $[18,23])$ provides the following description of $\widetilde{A}$ :

$$
\mathcal{D}(\widetilde{A})=\mathcal{D}\left(A_{N}\right) \dot{+}\left\{\left(I-C_{\widetilde{A}}\right) \eta_{i} \mid \forall \eta_{i} \in N_{i}\right\},
$$

where $C_{\widetilde{A}}=(\widetilde{A}-i I)(\widetilde{A}+i I)^{-1}$ is the Cayley transform of $\widetilde{A}$ and

$$
\widetilde{A}\left(u_{0}+\eta_{i}-C_{\widetilde{A}} \eta_{i}\right)=A u_{0}+i\left(I+C_{\widetilde{A}}\right) \eta_{i} \quad\left(u_{0} \in \mathcal{D}\left(A_{N}\right), \eta_{i} \in N_{i}\right) .
$$

The operator $A$ is described by the same formulas (with $C_{A}=(A-i I)(A+i I)^{-1}$ instead of $C_{\widetilde{A}}$ and the property of $A$ and $\widetilde{A}$ to be relatively prime with respect to $A_{N}$ means that $\left(C_{A}-C_{\widetilde{A}}\right) \uparrow_{N_{i}}$ is an invertible operator.

Taking into account that $A(A-z I)^{-1}$ maps $N$ onto $N_{z}$ and $\mathcal{R}\left(C_{\widetilde{A}}\left\lceil N_{i}\right)=N_{-i}\right.$, one can decompose any $f=u_{0}+\left(I-C_{\widetilde{A}}\right) \eta_{i} \in \mathcal{D}(\widetilde{A})$ as $f=u+\eta$, where

$$
u=u_{0}+i A^{-1}\left(I+C_{\widetilde{A}}\right) \eta_{i} \in \mathcal{D}(A), \quad \eta=(A+i I) A^{-1}\left(C_{A}-C_{\widetilde{A}}\right) \eta_{i} \in N
$$

and $\widetilde{A} f=A u$. These relations, enables one to rewrite the definition (2.12), (2.13) of $\widetilde{A}$ in the form (2.4), (2.5), where

$T \eta=-i P_{N}\left(I+C_{\widetilde{A}}\right)\left(C_{A}-C_{\widetilde{A}}\right)^{-1} A(A+i I)^{-1} \eta, \quad \eta \in \mathcal{D}(T)=(A+i I) A^{-1}\left(C_{A}-C_{\widetilde{A}}\right) N_{i}$.

The operator $T$ is well-defined (since $\operatorname{ker}\left(C_{A}-C_{\widetilde{A}}\right) \uparrow_{N_{i}}=\{0\}$ ), admissible (by Lemma 2.2), and self-adjoint (by Theorem 2.1). So, we show that $\widetilde{A}=A_{N, T}$.

It follows from Theorem 2.1 that the correspondence $\widetilde{A} \leftrightarrow\{N, T\}$ is a bijection between self-adjoint operators and pairs $\{N, T\}$, where $N$ is a subspace of $\mathcal{H}$ and $T$ is an admissible self-adjoint operator in $N$. Theorem 2.2 is proved.

REMARK 1. Theorem 2.2 can be also proved with the use of [21], where a description of all self-adjoint operators $\widetilde{A}$ has been obtained with the help of parameters $\{M, \gamma\}$ appearing in the Krein's resolvent formula relating $\widetilde{A}$ and $A$. Here $M=N_{-i}=\{\eta \in \mathcal{H} \mid((A+$ $i I) u, \eta)=0, A u=\widetilde{A} u\}$ is a defect subspace of $\widetilde{A} \wedge A$ and the self-adjoint operator $\gamma$ in $M$ is determined by the relation

$$
U=I-2 i(\gamma+i I)^{-1} P_{M},
$$

where the unitary operator $U$ has the form

$$
U=\left[(A-i I)(A+i I)^{-1}\right]\left[(\tilde{A}+i I)(\tilde{A}-i I)^{-1}\right] .
$$

Let us show that the equation

$$
(\widetilde{A}-i I)^{-1}=(A-i I)^{-1}-A(A-i I)^{-1}\left(Q_{0}(i)+T\right)^{-1} P_{N} A(A-i I)^{-1}
$$

has a unique solution $T$, where $T$ is self-adjoint in $N$ and $Q_{0}(i)=i P_{N} A(A-i I)^{-1} P_{N}$. Indeed, since $(\widetilde{A}+i I)(\widetilde{A}-i I)^{-1}=I+2 i(\widetilde{A}-i I)^{-1}$ by substituting (2.16) into (2.15) we 
have

$$
U=I-2 i A(A+i I)^{-1}\left(Q_{0}(i)+T\right)^{-1} P_{N} A(A-i I)^{-1} .
$$

Equating the right-hand sides of (2.14) and (2.17), enables one to establish an explicit expression of $T$ through $\gamma$ :

$$
T=P_{N} A(A-i I)^{-1} P_{M} \gamma P_{M} A(A+i I)^{-1} P_{N}+P_{N}\left(A+A^{-1}\right)^{-1} P_{N} .
$$

Since $\gamma$ is self-adjoint in $M=A(A+i I)^{-1} N$, relation (2.18) shows that $T$ is self-adjoint in $N$ and $\mathcal{D}(T)=(A+i I) A^{-1} \mathcal{D}(\gamma)$. It follows from (2.7) and (2.16) that $\left(A_{N, T}-i I\right)^{-1}=$ $(\widetilde{A}-i I)^{-1}$, i.e., $\widetilde{A}=A_{N, T}$.

Many properties of $A_{N, T}$ can be easily characterized through the parameters $N$ and $T$.

Proposition 2.2. Let $A_{N, T}$ be defined by (2.4), (2.5). Then:

(i) The zero operator $T=0$ is admissible for any choice of $N$. The corresponding operator $A_{N, 0}$ coincides with the Krein-von Neumann extension ${ }^{2}$ of $A_{N}$.

(ii) The operator $A_{N, T}$ is boundedly invertible if and only if $T$ is boundedly invertible in $N$. In that case $A_{N, T}^{-1}=A^{-1}-T^{-1} P_{N}$.

(iii) The operator $A_{N, T}$ is a finite rank perturbation of $A$ if and only if $\operatorname{dim} N<\infty$.

PROOF. Since $A \geq I$ the condition (2.6) provides the admissibility of the zero operator $T=0$ for any choice of $N$. The corresponding operator $A_{N, 0}$ has the domain $\mathcal{D}\left(A_{N, 0}\right)=$ $\mathcal{D}\left(A_{N}\right) \dot{+} N$ (by (2.8)). Employing Corollary 3 in [5], we derive that $A_{N, 0}$ coincides with the Krein-von Neumann extension of $A_{N}$.

Since $A \geq 1$, formulas (2.4), (2.5) imply that $0 \in \rho\left(A_{N, T}\right) \Leftrightarrow 0 \in \rho(T)$ and $A_{N, T}^{-1}=$ $A^{-1}-T^{-1} P_{N}$.

A self-adjoint operator $A_{N, T}$ is a finite rank perturbation of $A$ if the resolvent difference $\theta_{z}=\left(A_{N, T}-z I\right)^{-1}-(A-z I)^{-1}$ is a finite rank operator (i.e., $\left.\operatorname{dim} \mathcal{R}\left(\theta_{z}\right)<\infty\right)$ for at least one $z \in \rho(A) \cap \rho\left(A_{N, T}\right)$ [16]. Since $0 \in \rho\left(A(A-z I)^{-1}\right)$, the relation (2.7) yields $\operatorname{dim} \mathcal{R}\left(\theta_{z}\right)=\operatorname{dim} N$. Proposition 2.2 is proved.

2.3. BVS approach. As was mentioned above, the symmetric operator $A_{N}$ is not necessarily densely defined and the description of self-adjoint extensions $A_{N, T} \supset A_{N}$ cannot be obtained directly by methods of the extension theory of densely defined operators. An approach presented in this subsection allows one to avoid this inconvenience and to consider $A_{N, T}$ as an extension of a densely defined symmetric operator. Precisely, under the assumptions:

(1) $N_{r}=N \cap \mathcal{D}(A)$ is a closed subspace in $\mathcal{H}$;

(2) there exists a bounded self-adjoint operator $R$ in $N$ satisfying the condition

$$
R \eta_{r}=P_{N} A \eta_{r}, \quad \forall \eta_{r} \in N_{r}
$$

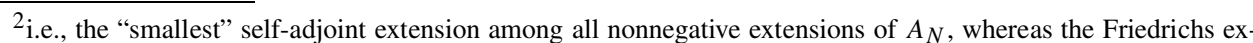
tension of $A_{N}$ is the "greatest" one, see [5] for details.
} 
and such that $(T-R)^{-1}$ is a bounded operator in $N$,

we construct a closed densely defined symmetric operator $A_{N, T \text {, min }}$ such that $A_{N} \subset$ $A_{N, T, \min } \subset A_{N, T}$ and describe $A_{N, T}$ in terms of BVS of $A_{N, T \text {, min }}^{*}$ (cf. Proposition 2.1).

It follows from (1) that the space $N$ admits the decomposition $N=N_{r} \oplus N_{s}$ into its singular $N_{s}=N \ominus N_{r}$ and regular $N_{r}=N \cap \mathcal{D}(A)$ parts with respect to $A$.

With respect to the decomposition $N=N_{r} \oplus N_{s}$, the operator $R$ can be presented as an operator-valued matrix $\left(\begin{array}{ll}R_{r r} & R_{r s} \\ R_{s r} & R_{s s}\end{array}\right)$. Here $R_{r r}=P_{N_{r}} A P_{N_{r}}, R_{s r}=P_{N_{s}} A P_{N_{r}}$ (by (2.19)), and $R_{r s}=R_{s r}^{*}$. The choice of $R_{s s}$ in $N_{s}$ is restricted by the condition of $(T-R)^{-1}$ to be bounded.

Since $\mathcal{D}(A)+N=\mathcal{D}(A) \dot{+} N_{s}$, the definition (2.4), (2.5) of $A_{N, T}$ can be rewritten with the use of $R$

$$
A_{N, T} f=A_{N, T}\left(v+\eta_{s}\right)=A v-A P_{N_{r}} \eta, \quad v \in \mathcal{D}(A), \quad \eta_{s} \in N_{s},
$$

where $\eta \in N$ and $\eta_{s}=P_{N_{s}} \eta$ satisfy the boundary condition

$$
(T-R) \eta=-\left(P_{N} A v+R \eta_{s}\right) .
$$

By analogy with (2.20) and (2.21), we define an operator $A_{N, T, \max }$ in $\mathcal{D}(A) \dot{+} N_{s}$ by the formulas

$$
A_{N, T, \max } f=A_{N, T, \max }\left(v+\eta_{s}\right)=A v-A P_{N_{r}} \xi, \quad v \in \mathcal{D}(A), \quad \eta_{s} \in N_{s},
$$

where $\xi \in N$ is determined for $f=v+\eta_{s}$ by the relation (cf. (2.21))

$$
(T-R) \xi=-\left(P_{N} A v+R \eta_{s}\right) .
$$

It follows from the definition of $A_{N, T, \text { max }}$ that $\mathcal{D}\left(A_{N, T, \max }\right) \subseteq \mathcal{D}(A) \dot{+} N_{s}$.

THEOREM 2.3. If assumptions (1), (2) hold, then the operator $A_{N, T, \max }$ possesses the following properties:

i) $\mathcal{D}\left(A_{N, T, \max }\right)=\mathcal{D}(A) \dot{+} N_{s}$.

ii) The abstract Green identity

$$
\left(A_{N, T, \max } f, g\right)-\left(f, A_{N, T, \max } g\right)=\left(\Gamma_{1} f, \Gamma_{0} g\right)_{N_{s}}-\left(\Gamma_{0} f, \Gamma_{1} g\right)_{N_{s}},
$$

$\left(f, g \in \mathcal{D}\left(A_{N, T, \max }\right)\right)$ holds, where

$$
\Gamma_{0} f=\Gamma_{0}\left(v+\eta_{s}\right)=P_{N_{s}}\left(A v+R \eta_{s}\right), \quad \Gamma_{1} f=\Gamma_{1}\left(v+\eta_{s}\right)=\xi_{s}-\eta_{s},
$$

$v \in \mathcal{D}(A), \eta_{s} \in N_{s}$, and $\xi_{s}=P_{N_{s}} \xi$, where $\xi \in N$ is determined by the relation (2.23).

iii) The map $\left(\Gamma_{0}, \Gamma_{1}\right): \mathcal{D}\left(A_{N, T, \max }\right) \rightarrow N_{s} \oplus N_{s}$ is surjective.

iv) The operator $A_{N, T, \min }=A_{N, T, \max }\left\lceil\mathcal{D}\left(A_{N, T, \min }\right)\right.$,

$$
\mathcal{D}\left(A_{N, T, \min }\right)=\left\{f \in \mathcal{D}(A) \dot{+} N_{s} \mid \Gamma_{0} f=\Gamma_{1} f=0\right\}
$$

is a closed symmetric densely defined operator in $\mathcal{H}$ and $A_{N, T, \min }^{*}=A_{N, T, \max }$. 
v) The triple $\left(N_{s}, \Gamma_{0}, \Gamma_{1}\right)$, where $\Gamma_{i}$ are determined by (2.25) is a BVS of $A_{N, T, \max }=$ $A_{N, T, \min }^{*}$

Proof. Since $(T-R)^{-1}$ is bounded, the relation (2.23) immediately implies i).

To establish the Green identity for $A_{N, T, \max }$, we denote $f^{i}=v^{i}+\eta_{s}^{i}\left(v^{i} \in \mathcal{D}(A)\right.$, $\left.\eta_{s}^{i} \in N_{s}, \quad i=1,2\right)$ and consider the corresponding $\xi^{i} \in \mathcal{D}(T)$ that are determined by (2.23). It follows from (2.22), (2.23) that

$$
\begin{aligned}
\left(A_{N, T, \max } f^{1}, f^{2}\right) & -\left(f^{1}, A_{N, T, \max } f^{2}\right)=\left(A v^{1}, \eta_{s}^{2}-\xi_{s}^{2}\right)-\left(\eta_{s}^{1}-\xi_{s}^{1}, A v^{2}\right) \\
& +\left(\eta_{s}^{1}, A P_{N_{r}} \xi^{2}\right)-\left(A P_{N_{r}} \xi^{1}, \eta_{s}^{2}\right)+\left(A v^{1}, \xi^{2}\right)-\left(\xi^{1}, A v^{2}\right) .
\end{aligned}
$$

Using the self-adjointness of $T$ and $R$ and the relation (2.23), we get $\left(A v^{1}, \xi^{2}\right)-$ $\left(\xi^{1}, A v^{2}\right)=\left(-(T-R) \xi^{1}-R \eta_{s}^{1}, \xi^{2}\right)-\left(\xi^{1},-(T-R) \xi^{2}-R \eta_{s}^{2}\right)=\left(R \xi^{1}, \eta_{s}^{2}\right)-\left(\eta_{s}^{1}, R \xi^{2}\right)$. Substituting the obtained expression into (2.27) and taking (2.19) into account, we establish (2.24).

To prove iii) it suffices to verify that

$$
\Gamma_{1} \mathcal{D}\left(A_{N, T, \max }\right)=N_{s} \quad \text { and } \quad\left(\Gamma_{0}, \Gamma_{1}\right) \mathcal{D}=N_{s} \oplus\{0\}
$$

for some set $\mathcal{D} \subset \mathcal{D}\left(A_{N, T, \max }\right)$. Indeed, for any $f=-A^{-1} R \eta_{s}+\eta_{s}$, relation (2.23) gives $\xi=0$ and hence, $\Gamma_{1} f=-\eta_{s}$. So, the first relation in (2.28) is true.

Let $w_{s} \in N_{s}$. Since $T-R$ is boundedly invertible in $N,(T-R) \xi=w_{s}$ for an element $\xi \in N$. Put $\eta_{s}=\xi_{s}$, and $v=-A^{-1}\left(w_{s}+R \eta_{s}\right)$. Then $\Gamma_{1}\left(v+\eta_{s}\right)=\xi_{s}-\eta_{s}=0$ and $\Gamma_{0}\left(v+\eta_{s}\right)=P_{N_{s}}\left(A v+R \eta_{s}\right)=-w_{s}$ that gives the second relation in (2.28) for $\mathcal{D}=\left\{v+\eta_{s}\right\}$.

Let us prove iv). It follows from (2.24) and (2.26) that $A_{N, T, \min }$ is a symmetric operator

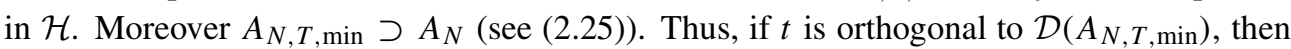
$t \perp \mathcal{D}\left(A_{N}\right)$ and hence, $t=A w_{r}$, where $w_{r} \in N_{r}$. Denote by $\xi$ the solution of the equation $(T-R) \xi=w_{r}$ and put $\eta_{s}=\xi_{s}=P_{N_{s}} \xi, v=-A^{-1}\left(w_{r}+R \eta_{s}\right)$. Then $\Gamma_{1}\left(v+\eta_{s}\right)=$ $\xi_{s}-\eta_{s}=0, \Gamma_{0}\left(v+\eta_{s}\right)=P_{N_{s}}\left(A v+R \eta_{s}\right)=0$. Thus $f=v+\eta_{s} \in \mathcal{D}\left(A_{N, T, \min }\right)$ and

$$
0=\left(f, A w_{r}\right)=\left(-A^{-1}\left(w_{r}+R \eta_{s}\right)+\eta_{s}, A w_{r}\right)=-\left(w_{r}, w_{r}\right)=0
$$

(here we use that $R=R^{*}$ and $P_{N} A w_{r}=R w_{r}$ ). Therefore $w_{r}=0$ and $A_{N, T \text {,min }}$ is a densely defined operator.

The adjoint $A_{N, T, \min }^{*}$ can be find by analogy with the description of $A_{N, T}^{*}$ in Theorem 2.1. Namely, we consider the equation $\left(A_{N, T, \min } f, g\right)=(f, w)$ for all $f \in \mathcal{D}\left(A_{N, T, \min }\right)$. The assumption $f \in \mathcal{D}\left(A_{N}\right)$ gives $g-A^{-1} w=\zeta \in N$. Hence $g=A^{-1} w+\zeta \in \mathcal{D}(A) \dot{+} N_{S}=$ $\mathcal{D}\left(A_{N, T, \max }\right)$. By (2.24), $\left(A_{N, T, \min } f, g\right)=\left(f, A_{N, T, \max } g\right)\left(\forall f \in \mathcal{D}\left(A_{N, T, \min }\right)\right)$. So, $w=$ $A_{N, T, \min }^{*} g$ and $A_{N, T, \min }^{*} g=A_{N, T, \max } g$.

The relation $A_{N, T, \max } \supset A_{N, T, \text { min }}$ implies $A_{N, T, \max }^{*} \subset A_{N, T, \max }$. The equality $A_{N, T, \max }^{*}=A_{N, T, \min }$ follows from the Green identity (2.24) and the property iii) of $\Gamma_{i}$. Thus $A_{N, T, \min }$ is a closed operator. 
By virtue of i)-iv), the triple $\left(N_{s}, \Gamma_{0}, \Gamma_{1}\right)$ is a BVS of $A_{N, T, \min }^{*}=A_{N, T, \max }$. Theorem 2.3 is proved.

THEOREM 2.4. If assumptions (1), (2) hold, then $A_{N, T}$ is a self-adjoint extension of the densely defined symmetric operator $A_{N, T, \min }$ and

$$
A_{N, T}=A_{N, T, \text { max }}\left\lceil\mathcal{D}\left(A_{N, T}\right), \quad \mathcal{D}\left(A_{N, T}\right)=\left\{f \in \mathcal{D}(A) \dot{+} N_{s} \mid \Gamma_{1} f=0\right\} .\right.
$$

Proof. If $(T-R)^{-1}$ exists, then $\operatorname{ker}(T-R)=\{0\}$ and, hence $\operatorname{ker}(T-R) \uparrow_{N_{r}}=\{0\}$. The last relation and (2.19) yield (2.6). So, $T$ is an admissible self-adjoint operator in $N$.

Let $A_{N, T}$ be a self-adjoint operator determined by (2.4), (2.5). Comparing (2.21) and (2.23) for any $f=v+\eta_{s} \in \mathcal{D}\left(A_{N, T}\right)$ one gets $\xi=\eta$. But then $\Gamma_{1} f=\xi_{s}-\eta_{s}=0$ and $A_{N, T} f=A_{N, T, \max } f$. Therefore, the self-adjoint operator $A_{N, T}$ is a restriction of $A_{N, T}^{\prime}=$ $A_{N, T, \max } \Upsilon_{\operatorname{ker} \Gamma_{1}}$. Since $\left(N_{s}, \Gamma_{0}, \Gamma_{1}\right)$ is a BVS of $A_{N, T, \max }$, the operator $A_{N, T}^{\prime}$ is self-adjoint [13]. Hence, $A_{N, T}=A_{N, T}^{\prime}$. Theorem 2.4 is proved.

In Theorems 2.3, 2.4 it is assumed that $B=(T-R)^{-1}$ is a bounded self-adjoint operator in $N$. This allows one to exclude $\xi$ in (2.22), (2.23) and to establish the following explicit expression for $A_{N, T, \max }$ :

$$
A_{N, T, \max } f=A_{N, T, \max }\left(v+\eta_{s}\right)=A v+A P_{N_{r}} B P_{N}\left(A v+R \eta_{s}\right)
$$

for all $v \in \mathcal{D}(A)$ and $\eta_{s} \in N_{s}$. Combining (2.29) with Theorem 2.4, one gets

THEOREM 2.5. Let B be a bounded self-adjoint operator in $N=N_{r} \oplus N_{s}, N_{r}=$ $N \cap \mathcal{D}(A)$ is a closed subspace, and $\operatorname{ker} B=\{0\}$. Then the operator

$$
\widetilde{A} f=\widetilde{A}\left(v+\eta_{s}\right)=A v+A P_{N_{r}} B P_{N}\left(A v+R \eta_{s}\right),
$$

where elements $v \in \mathcal{D}(A)$ and $\eta_{s} \in N_{s}$ satisfy the boundary condition

$$
\eta_{s}+P_{N_{s}} B\left(P_{N} A v+R \eta_{s}\right)=0
$$

is self-adjoint in $\mathcal{H}$ and $\widetilde{A} \wedge A=A_{N}$.

Note that Theorem 2.5 can be proved without employing of previous theorems. To do this, it suffices to establish the Green identity for the operator $A_{N, T \text {,max }}$ defined by (2.29) with the boundary operators

$\Gamma_{0} f=\Gamma_{0}\left(v+\eta_{s}\right)=P_{N_{s}}\left(A v+R \eta_{s}\right), \quad \Gamma_{1} f=\Gamma_{1}\left(v+\eta_{s}\right)=-\eta_{s}-P_{N_{s}} B\left(P_{N} A v+R \eta_{s}\right)$ in the right-hand side and to verify that the triple $\left(N_{s}, \Gamma_{0}, \Gamma_{1}\right)$ is a BVS.

In a particular case where $N=N_{r}$, the following result can be easily proved.

THEOREM 2.6. Let $B$ be a bounded self-adjoint operator in $N \subset \mathcal{D}(A)$. Then the operator

$$
\widetilde{A}=A+A B P_{N} A, \quad \mathcal{D}(\widetilde{A})=\mathcal{D}(A)
$$

is self-adjoint in $\mathcal{H}$. The relation $\widetilde{A} \wedge A=A_{N}$ holds if and only if $\operatorname{ker} B=\{0\}$. 
REMARK 2. In this subsection, operators $A_{N, T}$ are studied under assumptions (1), (2) (see the introductory part of subsection). Their weakening requires an additional considerations. However they are immediately satisfied in a series of important particular cases. For instance, in the case of purely singular perturbations $N_{r}=\{0\}$, the condition (1) is trivial and (2) is obviously satisfied for any self-adjoint operator $T$. Another example deals with the case of finite rank perturbations. Here (1) is obvious and (2) is justified by the following assertion.

Proposition 2.3. Let $T$ be an admissible self-adjoint operator in $N=N_{r} \oplus N_{s}$ and $\operatorname{dim} N<\infty$. Then there exists a self-adjoint operator $R$ in $N$ satisfying condition (2.19) and such that $\operatorname{ker}(T-R)=\{0\}$.

PROOF. Let

$$
C=T-R=\left(\begin{array}{ll}
C_{r r} & C_{r s} \\
C_{s r} & C_{s s}
\end{array}\right), \quad C_{r r}=C_{r r}^{*}, \quad C_{s s}=C_{s s}^{*}, \quad C_{r s}=C_{s r}^{*}
$$

Since the property of $T$ to be admissible is equivalent the relation $\operatorname{ker} C_{r r} \cap \operatorname{ker} C_{s r}=\{0\}$, we complete the proof after the finding $C_{s s}$ such that $\operatorname{ker} C=\{0\}$.

Let us consider $C_{s s}=I_{s}+C_{s r}\left|C_{r r}\right|^{-1} P_{N_{1}} C_{r s}$, where $I_{s}$ is the identity operator in $N_{s}$ and $N_{1}=N_{r} \ominus \operatorname{ker} C_{r r}$. It is clear that $C_{r r}^{-1} P_{N_{1}}$ and $\left|C_{r r}\right|^{-1} P_{N_{1}}$ exist and hence, $C_{s s}$ is well-defined. Assume that $x=x_{r}+x_{s} \in \operatorname{ker} C$, i.e.,

$$
C_{r r} x_{r}+C_{r s} x_{s}=0, \quad C_{s r} x_{r}+C_{s s} x_{s}=0 .
$$

It follows from the first relation that $\left(C_{r s} x_{s}, x_{0}\right)=0, \forall x_{0} \in \operatorname{ker} C_{r r}$ and $x_{r}=\tilde{x}_{0}-$ $C_{r r}^{-1} P_{N_{1}} C_{r s} x_{s}, \tilde{x}_{0} \in \operatorname{ker} C_{r r}$. Substituting the obtained expression of $x_{r}$ into the second relation of (2.33) and multiplying it by $x_{s}$ we get

$$
\left(x_{s}, x_{s}\right)+\left(C_{s r}\left[\left|C_{r r}\right|^{-1}-C_{r r}^{-1}\right] P_{N_{1}} C_{r s} x_{s}, x_{s}\right)=0 .
$$

Here the second term is nonnegative ( since $C_{r s}=C_{s r}^{*}$ ) and hence, $x_{s}=0$. But then $C_{r r} x_{r}=0$ and $C_{s r} x_{r}=0$ (see (2.33)) i.e., $x_{r} \in \operatorname{ker} C_{r r} \cap \operatorname{ker} C_{s r}=\{0\}$. Therefore, $\operatorname{ker} C=\{0\}$. Proposition 2.3 is proved.

\section{Additive perturbations theory approach}

To describe self-adjoint perturbations $\widetilde{A}$ of $A$ as $A+V$, in general, one needs to use "potentials" $V$ acting in the scale of Hilbert spaces associated with $A$ ( $A$-scale).

We recall [7] that $A$-scale is the collection of Hilbert spaces $\mathcal{H}_{s} \subset \mathcal{H}=\mathcal{H}_{0} \subset \mathcal{H}_{-s} s>$ 0 , where $\mathcal{H}_{s}=\mathcal{D}\left(A^{s / 2}\right),\|u\|_{s}=\left\|A^{s / 2} u\right\|$ and $\mathcal{H}_{-s}$ is the completion of $\mathcal{H}_{0}$ with respect to $\|u\|_{-s}=\left\|A^{-s / 2} u\right\|$. We will use the notation $\mathbf{A}$ for the continuation of $A$ as an isometric mapping from $\mathcal{H}_{0}=\mathcal{H}$ onto $\mathcal{H}_{-2}$. Then the inner product in $\mathcal{H}$ can be extended to a pairing between $\mathcal{H}_{s}$ and $\mathcal{H}_{-s}$. In particular, for $u \in \mathcal{H}_{2}=\mathcal{D}(A)$ and $\psi \in \mathcal{H}_{-2}$, we have $\langle u, \psi\rangle=$ $\left(A u, \mathbf{A}^{-1} \psi\right)=\overline{\langle\psi, u\rangle}$. 
An operator $\mathbf{L}: \mathcal{H}_{s} \rightarrow \mathcal{H}_{-s}$ is called Hermitian if $\langle\mathbf{L} u, v\rangle=\langle u, \mathbf{L} v\rangle$ for all $u, v \in$ $\mathcal{D}(\mathbf{L}) \subset \mathcal{H}_{s}$.

Let $\mathbf{L}: \mathcal{H} \rightarrow \mathcal{H}_{-2}$. In that case, the operator $L=\mathbf{L}\lceil\mathcal{D}$, where $\mathcal{D}=\{u \in \mathcal{D}(\mathbf{L}) \mid \mathbf{L} u \in$ $\mathcal{H}$ \} is called the $\mathcal{H}$-part of $\mathbf{L}$ (or the restriction of $\mathbf{L}$ in $\mathcal{H}, L=\mathbf{L}\left\llcorner_{\mathcal{H}}\right.$ ). If $\mathbf{L} \neq \mathbf{L}\left\llcorner_{\mathcal{H}}\right.$, then the operator $\mathbf{L}$ is called singular in $\mathcal{H}$.

Obviously, that $\mathbf{A}\left\llcorner_{\mathcal{H}}=A\right.$ and $\mathbf{A}$ is a singular operator in $\mathcal{H}$.

PROPOSITION 3.1. Under the conditions of Theorem 2.5, we consider the singular operator

$$
\widetilde{\mathbf{A}} f=\mathbf{A} f+\mathbf{A} B P_{N}\left(A v+R \eta_{s}\right), \quad f=v+\eta_{s}, \quad v \in \mathcal{H}_{2}, \quad \eta_{s} \in N_{s}
$$

determined on $\mathcal{H}_{2} \dot{+} N_{s}$. Then the $\mathcal{H}$-part of $\widetilde{\mathbf{A}}$ coincides with the self-adjoint operator $\widetilde{A}$ in Theorem 2.5.

PROOF. Since

$$
\widetilde{\mathbf{A}} f=\widetilde{\mathbf{A}}\left(v+\eta_{s}\right)=\widetilde{A}\left(v+\eta_{s}\right)+\mathbf{A}\left[\eta_{s}+P_{N_{s}} B P_{N}\left(A v+R \eta_{s}\right)\right],
$$

where $\widetilde{A}$ is determined by (2.30), the condition $\widetilde{\mathbf{A}}\left(v+\eta_{s}\right) \in \mathcal{H}$ is equivalent to the boundary condition (2.31). Hence, $\widetilde{\mathbf{A}} \downarrow_{\mathcal{H}}=\widetilde{A}$. Proposition 3.1 is proved.

THEOREM 3.1. Let $\mathbf{V}: \mathcal{H}_{2} \rightarrow \mathcal{H}_{-2}$ be a bounded Hermitian operator and let $N=$ $\mathbf{A}^{-1} \mathcal{R}(\mathbf{V})=\mathcal{H} \ominus A \operatorname{ker} \mathbf{V}$ be a subspace of $\mathcal{H}$ such that $N=N_{r} \oplus N_{s}$, where $N_{r}=N \cap \mathcal{H}_{2}$. Assume that $R$ is a self-adjoint operator in $N$ satisfying the condition (2.19) and consider the extension (regularization)

$$
\mathbf{V}_{R} f=\mathbf{V}\left(u+A^{-1} R \eta_{s}\right), \quad f=u+\eta_{s}, \quad u \in \mathcal{H}_{2}, \quad \eta_{s} \in N_{s}
$$

of $\mathbf{V}$ onto $\mathcal{H}_{2} \dot{+} N_{s}$. Then the $\mathcal{H}$-part of the singular operator $\widetilde{\mathbf{A}}=\mathbf{A}+\mathbf{V}_{R}$ is a self-adjoint operator $\widetilde{A}=\widetilde{\mathbf{A}} L_{\mathcal{H}}$ in $\mathcal{H}$ and $\widetilde{A} \wedge A=A_{N}$.

PRoOF. The operator $B=\mathbf{A}^{-1} \mathbf{V} A^{-1} P_{N}$ is a bounded self-adjoint operator in $N$ and $\operatorname{ker} B=\{0\}$. It is easily to see that the singular operator $\widetilde{\mathbf{A}}=\mathbf{A}+\mathbf{V}_{R}$ coincides with the operator $\widetilde{\mathbf{A}}$ defined by (3.1). Using Proposition 3.1, we complete the proof of Theorem 3.1.

REMARK 3. The necessity of an extension (regularization) of an Hermitian singular "potential" $\mathbf{V}$ for the construction of self-adjoint realizations of $A+\mathbf{V}$ in $\mathcal{H}$ has been justified in [2] for the case of finite rank purely singular perturbations. For Schrödinger operators with point interactions, the ideology of the regularization of an initial expression to the wider domain of the formal adjoint operator is often used in physical works and it was inspired by Fermi's work [12], where a "pseudo-potential" was used for regularization.

If $\mathcal{R}(\mathbf{V}) \subset \mathcal{H}_{-1}$, i.e., $N \subset \mathcal{H}_{1}$, a singular operator $\mathbf{V}: \mathcal{H}_{2} \rightarrow \mathcal{H}_{-2}$ can be continuously extended onto $\mathcal{H}_{1}$ as a bounded Hermitian operator $\widetilde{\mathbf{V}}: \mathcal{H}_{1} \rightarrow \mathcal{H}_{-1}$. The operator $\widetilde{\mathbf{V}}$ is defined on $\mathcal{H}_{1} \supset \mathcal{H}_{2}+N_{s}$ and it is called natural extension of $\mathbf{V}$. In that case, the regularizing operator 
$R$ is determined uniquely by the equality $(R \eta, \widetilde{\eta})=\left(A^{1 / 2} \eta, A^{1 / 2} \xi\right), \forall \eta, \xi \in N \subset \mathcal{H}_{1}$ and it is called natural.

The next statement is a direct consequence of Theorem 3.1.

Proposition 3.2. Let $\widetilde{\mathbf{V}}: \mathcal{H}_{1} \rightarrow \mathcal{H}_{-1}$ be a bounded Hermitian operator. Then the $\mathcal{H}$-part of the singular operator $\widetilde{\mathbf{A}}=\mathbf{A}+\widetilde{\mathbf{V}}$ is a self-adjoint operator $\widetilde{A}=(\mathbf{A}+\widetilde{\mathbf{V}}) L_{\mathcal{H}}$ in $\mathcal{H}$.

REMARK 4. In [17] for purely singular perturbations of the form

$$
\widetilde{\mathbf{A}}=\mathbf{A}+\alpha \psi_{-1}\left\langle\cdot, \psi_{-1}\right\rangle, \quad \psi_{-1} \in \mathcal{H}_{-1} \backslash \mathcal{H}
$$

it has been shown that the Friedrichs extension of the corresponding minimal operator is determined by (3.2) with the coupling constant $\alpha=\infty$. Such a property can be generalized as follows.

Proposition 3.3. A purely singular perturbation $\widetilde{A}$ of A coincides with the Friedrichs extension $A_{F}$ of $\widetilde{A} \wedge A$ if and only if $N \subset \mathcal{H}_{1}$, where $N$ is the defect subspace of $\widetilde{A} \wedge A$. In this case, $\widetilde{A}=A_{F}=A_{N, T}$, where $T$ is determined by the relation

$$
(T \eta, \xi)=\left(A^{1 / 2} \eta, A^{1 / 2} \xi\right), \quad \forall \eta, \quad \xi \in N \subset \mathcal{H}_{1}
$$

and $A_{F}^{-1}=A^{-1}-P_{N}^{\mathcal{H}_{1}} A^{-1}$, where $P_{N}^{\mathcal{H}_{1}}$ is an orthoprojector in the Hilbert space $\mathcal{H}_{1}$ onto $N$.

Proof. Since $\widetilde{A}$ and $A$ are relatively prime extensions of the symmetric operator $A_{N}=$ $\widetilde{A} \wedge A$ with the defect subspace $N$, the general properties of the Friedrichs extension yield that $\widetilde{A}=A_{F} \Leftrightarrow N \subset \mathcal{H}_{1}$ (see e.g., [6]). The Friedrichs extension $A_{F}$ is distinguished by the condition $\left(A_{F} f, g\right)=\left(A^{1 / 2} f, A^{1 / 2} g\right)\left(\forall f, g \in \mathcal{D}\left(A_{F}\right)\right)$ among all self-adjoint extensions $A_{N, T}$ of $A_{N}$. Using (2.8) and (2.9) we rewrite this condition as $(T \eta, \xi)=\left(A^{1 / 2} \eta, A^{1 / 2} \xi\right)$ $\left(\forall \eta, \xi \in N \subset \mathcal{H}_{1}\right)$.

By virtue of assertion (ii) of Proposition 2.2, $A_{F}^{-1}=A^{-1}-T^{-1} P_{N}$. Using (3.3), it is easy to show that $T P_{N}^{\mathcal{H}_{1}} A^{-1}=P_{N}$ that gives $\widetilde{A}^{-1}=A^{-1}-P_{N}^{\mathcal{H}_{1}} A^{-1}$. Indeed, $\left(T P_{N}^{\mathcal{H}_{1}} A^{-1} f, \eta\right)=$ $\left(A^{1 / 2} P_{N}^{\mathcal{H}_{1}} A^{-1} f, A^{1 / 2} \eta\right)=\left(P_{N}^{\mathcal{H}_{1}} A^{-1} f, \eta\right) \mathcal{H}_{1}=\left(A^{-1} f, \eta\right)_{\mathcal{H}_{1}}=\left(P_{N} f, \eta\right)$. Proposition 3.3 is proved.

REMARK 5. Under the conditions of Proposition 3.3, the natural regularizing operator $R$ coincides with $T$. This means that $B=(T-R)^{-1}$ does not exist. In this case, from the physical point of view, infinite value of the coupling operator-parameter $B$ can be used.

REMARK 6. The non-uniqueness of the regularizing operator $R$ (up to the choice of $R_{s s}$ ) is useful for the description of all finite rank perturbations in terms of singular additive perturbations (see Proposition 3.1 and Remark 2) and for the preservation of initial symmetries of the potential $\mathbf{V}$ in its regularization $\mathbf{V}_{R}$ (see e.g., $[2,15]$ ). 


\section{The resolvent approximation}

Proposition 4.1. Let a finite dimensional subspace $N$ be fixed and let $T_{i}$ be a sequence of admissible operators that converges to an admissible operator $T$ in $N$. Then $A_{N, T_{i}}$ converges to $A_{N, T}$ in the norm resolvent sense.

Proof. To justify that $A_{N, T_{i}} \rightarrow A_{N, T}$ in the norm resolvent sense it suffices to show that $\left\|\left(A_{N, T_{i}}-z I\right)^{-1}-\left(A_{N, T}-z I\right)^{-1}\right\| \rightarrow 0$ for a fixed $z \in \mathbb{C} \backslash \mathbb{R}$. By virtue of (2.7), the latter relation is equivalent to

$$
\left\|\left[\left(Q_{0}(z)+T_{i}\right)^{-1}-\left(Q_{0}(z)+T\right)^{-1}\right] P_{N}\right\| \rightarrow 0 .
$$

To verify (4.1), we fix an orthonormal basis $\left\{\eta_{j}\right\}_{1}^{n}$ in $N$ and consider matrices $\Theta_{i}=$ $\left(\theta_{k l}^{i}\right)_{k, l=1}^{n}$ and $\Theta=\left(\theta_{k l}\right)_{k, l=1}^{n}$ that correspond to the operators $Q_{0}(z)+T_{i}$ and $Q_{0}(z)+T$, respectively, in the basis $\left\{\eta_{j}\right\}_{1}^{n}$.

Since $T_{i} \rightarrow T$, the operators $Q_{0}(z)+T_{i}$ converge to $Q_{0}(z)+T$ as $i \rightarrow \infty$. In this case, $\theta_{k l}^{i} \rightarrow \theta_{k l}$ for any $1 \leq k, l \leq n$ [14]. Hence, $\operatorname{det} \Theta_{i}$ converges to $\operatorname{det} \Theta$, where $\operatorname{det} \Theta$ is a non-zero number (since $Q_{0}(z)+T$ is invertible). This means that $\frac{1}{\operatorname{det} \Theta_{i}} \rightarrow \frac{1}{\operatorname{det} \Theta}$ and any entry $\chi_{k l}^{i}$ of the inverse matrix $\Theta_{i}^{-1}$ converges to the corresponding entry $\chi_{k l}$ of $\Theta^{-1}$. This property is equivalent to (4.1) [14]. Proposition 4.1 is proved.

THEOREM 4.1. For any finite rank self-adjoint perturbation $A_{N, T}$ there exists a sequence $A_{N_{i}, T_{i}}=A+V_{i}\left(V_{i}=A\left(T-P_{N_{i}} A\right)^{-1} P_{N_{i}} A\right)$ of regular finite rank perturbations that converges to $A_{N, T}$ in the norm resolvent sense, as $i \rightarrow \infty$.

Proof. Let $A_{N, T}$ be a singular finite rank perturbation of $A$ and let $\left\{\eta_{j}\right\}_{1}^{n}$ be an orthonormal basis in $N$. Since $\mathcal{D}(A)$ is dense in $\mathcal{H}$, any vector $\eta_{j}$ of the basis can be approximated by elements from $\mathcal{D}(A)$. Combining this with the Gramm-Schmidt orthonormalization procedure it is easy to establish the existence of an orthonormal system $\left\{\eta_{j}^{\varepsilon}\right\}_{1}^{n}$ of elements from $\mathcal{D}(A)$ such that

$$
\left\|\eta_{j}-\eta_{j}^{\varepsilon}\right\|<\varepsilon, \quad 1 \leq j \leq n, \quad \forall \varepsilon>0
$$

Let us choose a decreasing sequence $\varepsilon_{i} \rightarrow 0$ and let $\left\{\eta_{j}^{\varepsilon_{i}}\right\}_{1}^{n}$ be the corresponding orthonormal system satisfying (4.2) for $\varepsilon=\varepsilon_{i}$. Obviously, the linear span $N_{i}$ of $\left\{\eta_{j}^{\varepsilon_{i}}\right\}_{1}^{n}$ is contained in $\mathcal{D}(A)$ and the sequence of subspaces $\left\{N_{i}\right\}$ approximates $N$ in the sense of (4.2).

To define a sequence $\left\{T_{i}\right\}$ of admissible operators in $N_{i}$ we fix the matrix $\mathcal{T}=\left(t_{k l}\right)_{k, l=1}^{n}$ corresponding to the given operator $T$ in the basis $\left\{\eta_{j}\right\}_{1}^{n}$ and determine an operator $T_{i}$ having in $N_{i}$ the same matrix representation $\mathcal{T}$ but with respect to the basis $\left\{\eta_{j}^{\varepsilon_{i}}\right\}_{1}^{n}$. Of course, the operator $T_{i}$ constructed in such a way need not be admissible (i.e. may happen that $\operatorname{ker}\left(T_{i}-\right.$ $P_{N_{i}} A$ ) is not trivial). However, the admissibility of $T_{i}$ is easily achieved by the replacement of $T_{i}$ by $T_{i}-\alpha I$, where $\alpha>0$ can be chosen less than an arbitrary fixed positive number. 
In what follows, for definiteness, we will assume that the entries of the matrix $\mathcal{T}_{i}=$ $\left(t_{k l}^{i}\right)_{k, l=1}^{n}$ corresponding to the admissible operator $T_{i}$ in the basis $\left\{\eta_{j}^{\varepsilon_{i}}\right\}_{1}^{n}$ satisfy the relation

$$
\left|t_{k l}-t_{k l}^{i}\right|<\varepsilon_{i}, \quad 1 \leq k, l \leq n, \quad \forall i \in \mathbb{N}
$$

The regular subspaces $N_{i}$ and admissible operators $T_{i}$ constructed above allow one to determine with the help of (2.32) a sequence $\left\{A_{N_{i}, T_{i}}\right\}$ of finite rank regular perturbations of A.

By analogy with the proof of Proposition 4.1 we conclude that the convergence $A_{N_{i}, T_{i}} \rightarrow$ $A_{N, T}$ for $i \rightarrow \infty$ in the norm resolvent sense is equivalent to the convergence

$$
\left\|\left(Q_{0}^{i}(z)+T_{i}\right)^{-1} P_{N_{i}}-\left(Q_{0}(z)+T\right)^{-1} P_{N}\right\| \rightarrow 0, \quad i \rightarrow \infty,
$$

where $P_{N_{i}}$ are orthoprojectors onto $N_{i}$ in $\mathcal{H}, z \in \mathbb{C} \backslash \mathbb{R}$ is fixed, and $Q_{0}^{i}(z)=z P_{N_{i}} A(A-$ $z I)^{-1}$.

Let $f \in \mathcal{H}$. Then $\left(Q_{0}(z)+T\right)^{-1} P_{N} f=\left(\eta_{1}, \ldots, \eta_{n}\right) \Theta^{-1} \operatorname{col}\left(\left(f, \eta_{1}\right), \ldots,\left(f, \eta_{n}\right)\right)$ and

$$
\left(Q_{0}^{i}(z)+T_{i}\right)^{-1} P_{N_{i}} f=\left(\eta_{1}^{\varepsilon_{i}}, \ldots, \eta_{n}^{\varepsilon_{i}}\right) \Theta_{i}^{-1} \operatorname{col}\left(\left(f, \eta_{1}^{\varepsilon_{i}}\right), \ldots,\left(f, \eta_{n}^{\varepsilon_{i}}\right)\right) .
$$

Here $\Theta=\left(\theta_{k l}\right)_{k, l=1}^{n}$ and $\Theta_{i}=\left(\theta_{k l}^{i}\right)_{k, l=1}^{n}$ are the matrix representations of $Q_{0}(z)+T$ and $Q_{0}^{i}(z)+T_{i}$ in the basis $\left\{\eta_{j}\right\}_{1}^{n}$ and $\left\{\eta_{j}^{\varepsilon_{i}}\right\}_{1}^{n}$, respectively.

It follows from (4.2) and (4.3) that the entries $\theta_{k l}^{i}$ of $\Theta_{i}$ converge to the corresponding entries $\theta_{k l}$ of $\Theta$. Using this fact and arguing as in the proof of Proposition 4.1, we conclude that the entries $\chi_{k l}^{i}$ of the inverse matrix $\Theta_{i}^{-1}$ converge to the corresponding entries $\chi_{k l}$ of $\Theta^{-1}$ as $i \rightarrow \infty$.

Let us fix an arbitrarily small $\varepsilon>0$ and choose $m \in \mathbb{N}$ such that the inequalities $\varepsilon_{i}<$ $\varepsilon$ and $\left|\chi_{k l}-\chi_{k l}^{i}\right|<\varepsilon, 1 \leq k, l \leq n$ hold for all $i>m$. Using (4.2) and (4.5) it is easy to verify that

$$
\left\|\left(Q_{0}^{i}(z)+T_{i}\right)^{-1} P_{N_{i}} f-\left(Q_{0}(z)+T\right)^{-1} P_{N} f\right\|<\left(3 n^{2} \chi\right) \varepsilon\|f\|, \quad \forall i>m,
$$

where $\chi=\max _{1 \leq k, l \leq n}\left|\chi_{k l}\right|$. Since $f$ is arbitrary element from $\mathcal{H}$, the upper bound (4.6) implies (4.4). Theorem 4.1 is proved.

\section{Finite rank perturbations of the Schrödinger operators}

5.1. One point interaction in $\mathbb{R}^{3}$. Let us consider the Schrödinger operator with one point interaction determined by the expression $-\Delta+I+b\langle\cdot, \delta\rangle \delta(b \in \mathbb{R})$, where $\delta$ is the Dirac $\delta$-function (with support at 0 ).

In this case, the free Schrödinger operator has the form $A=-\Delta+I, \mathcal{D}(A)=\mathcal{H}_{2}=$ $W_{2}^{2}\left(\mathbb{R}^{3}\right)$, where $W_{2}^{2}\left(\mathbb{R}^{3}\right)$ is the Sobolev space. The operator $A$ acts in $L_{2}\left(\mathbb{R}^{3}\right)$. The dual space $\mathcal{H}_{-2}$ coincides with $W_{2}^{-2}\left(\mathbb{R}^{3}\right)$ and $V=b\langle\cdot, \delta\rangle \delta(x)$ is a symmetric singular operator acting from $\mathcal{H}_{2}$ in $\mathcal{H}_{-2}$. 
Obviously, ker $V=\left\{u(x) \in W_{2}^{2}\left(\mathbb{R}^{3}\right) \mid u(0)=0\right\}$. The restriction of $A$ onto ker $V$ determines a symmetric densely defined operator $A_{N}, \mathcal{D}\left(A_{N}\right)=\operatorname{ker} V($ see $(1.1)$ ), where the defect subspace $N=L_{2}\left(\mathbb{R}^{3}\right) \ominus A \operatorname{ker} V$ coincides with the linear span of $\frac{e^{-|x|}}{|x|}$.

To define a regularization $\mathbf{V}_{R}$ on $\mathcal{D}\left(A_{N}^{*}\right)=W_{2}^{2}\left(\mathbb{R}^{3}\right) \dot{+} N$, we consider the linear functionals $\Gamma_{i}: \mathcal{D}\left(A_{N}^{*}\right) \rightarrow \mathbb{C}(i=0,1)$

$$
\Gamma_{1} f=f_{s}:=4 \pi \lim _{|x| \rightarrow 0}|x| f(x), \quad \Gamma_{0} f=f_{r}:=\lim _{|x| \rightarrow 0}\left(f(x)-\frac{f_{s}}{4 \pi|x|}\right)
$$

and define the following extension of $V$ onto $\mathcal{D}\left(A_{N}^{*}\right)$ :

$$
\mathbf{V}_{R} f(x)=b f_{r} \delta(x), \quad \forall f \in \mathcal{D}\left(A_{N}^{*}\right)=W_{2}^{2}\left(\mathbb{R}^{3}\right) \dot{+} N .
$$

It is interesting to note that the well known pseudo-potential

$$
V_{F} f(x)=b\left[\frac{\partial}{\partial|x|}|x| f(x)\right]_{|x|=0} \delta(x)
$$

suggested by Fermi [12] in 1936 determines exactly the same regularization $\mathbf{V}_{R}$.

The operator $\mathbf{A}=-\Delta+I$ is determined in the $A$-scale and it maps $L_{2}\left(\mathbb{R}^{3}\right)$ onto $W_{2}^{-2}\left(\mathbb{R}^{3}\right)$, where the right-hand side is understood in the distributional sense, i.e.,

$$
\mathbf{A} f=(-\Delta+I) f=-(\Delta f)(x)+f(x)+f_{s} \delta(x), \quad f \in W_{2}^{2}\left(\mathbb{R}^{3}\right) \dot{+} N .
$$

Here the symbol $-(\Delta f)(x)$ means the action of $-\Delta$ in the point-wise sense except for $x=0$.

The $L_{2}\left(\mathbb{R}^{3}\right)$-part of $\mathbf{A}+\mathbf{V}_{R}$ is a self-adjoint operator $\widetilde{A}$ in $L_{2}\left(\mathbb{R}^{3}\right)$ and it can be considered as a model of the Schrödinger operator with one point interaction determined by the singular potential $V$. The operator $\widetilde{A}$ is a purely singular perturbation of $A$.

Since $f \in \mathcal{D}(\widetilde{A}) \Leftrightarrow\left(\mathbf{A}+V_{R}\right) f \in L_{2}\left(\mathbb{R}^{3}\right)$, relations (5.2) and (5.3) imply

$$
\mathcal{D}(\tilde{A})=\left\{f \in W_{2}^{2}\left(\mathbb{R}^{3}\right) \dot{+} N \mid f_{s}+b f_{r}=0\right\}
$$

and $\widetilde{A} f(x)=-(\Delta f)(x)+f(x)$.

To get a description of $\widetilde{A}$ in the extension theory framework we note that the triple $\left(\mathbb{C}, \Gamma_{0}, \Gamma_{1}\right)$, where $\Gamma_{i}$ are determined by (5.1) is a BVS of $A_{N}$. In terms of this BVS, the operator $\widetilde{A}$ can be described as the restriction of $A_{N}^{*}$ onto $\mathcal{D}(\widetilde{A})=\left\{f \in \mathcal{D}\left(A_{N}^{*}\right) \mid-b \Gamma_{0} f=\Gamma_{1} f\right\}$.

5.2. Nonlocal point interaction in $\mathbb{R}$. Let us consider a Schrödinger operator that is determined by the expression $-\frac{d^{2}}{d x^{2}}+I+V$ with a singular symmetric potential

$$
V=b_{11}\langle\cdot, \delta\rangle \delta+b_{12}(\cdot, q) \delta+b_{21}\langle\cdot, \delta\rangle q+b_{22}(\cdot, q) q .
$$

Here the coefficients $b_{i j} \in \mathbb{C}$ form an Hermitian matrix $\mathcal{B}$.

The nonperturbed operator $A$ has the form $A=-d^{2} / d x^{2}+I, \mathcal{D}(A)=W_{2}^{2}(\mathbb{R})=\mathcal{H}_{2}$ and the defect subspace $N \subset L_{2}(\mathbb{R})$ coincides with the linear span of functions $\eta_{1}(x)=$ 
$A^{-1} \delta=\frac{1}{2} e^{-|x|}$ and $\eta_{2}(x)=A^{-1} q(x)$. Since $\eta_{2}(x) \in \mathcal{D}(A)$, the corresponding symmetric operator $A_{N}$ is non-densely defined.

Self-adjoint extensions of $A_{N}$ are examples of mixed singular perturbations of $A$. To describe such type of extensions with the help of additive perturbations $A+V$, we need to construct some extension (regularization) of $V$ onto $W_{2}^{2}(\mathbb{R})+N$. This is a simple problem because $W_{2}^{2}(\mathbb{R})+N \subset \mathcal{H}_{1}$ and the delta function $\delta$ admits the extension by continuity onto $W_{2}^{2}(\mathbb{R})+N$ defined by the formula $\langle f, \delta\rangle=f(0)\left(f \in W_{2}^{2}(\mathbb{R})+N\right)$. As a result we get

$$
\mathbf{V}_{R} f=b_{11} f(0) \delta(x)+b_{12}(f, q) \delta(x)+b_{21} f(0) q(x)+b_{22}(f, q) q(x) .
$$

The operator $\mathbf{A}=-\frac{d^{2}}{d x^{2}}+I$ maps $L_{2}(\mathbb{R})$ onto $W_{2}^{-2}(\mathbb{R})$, where the derivative is understood in the distributional sense, i.e.,

$$
-\frac{d^{2}}{d x^{2}} f(x)=-f^{\prime \prime}(x)-f_{s}^{\prime} \delta(x)-f_{s} \delta^{\prime}(x), \quad \forall f \in W_{2}^{2}(\mathbb{R} \backslash\{0\}),
$$

where $f_{s}=f(+0)-f(-0), f_{s}^{\prime}=f^{\prime}(+0)-f^{\prime}(-0)$, and $f^{\prime \prime}(x)$ means the ordinary second derivative for $x \neq 0$.

Since any function $f \in W_{2}^{2}(\mathbb{R})+N$ is continuous at $x=0$, the formula (5.5) takes the form: $\mathbf{A} f=-f^{\prime \prime}(x)+f-f_{s}^{\prime} \delta(x), \forall f \in W_{2}^{2}(\mathbb{R})+N$. Therefore,

$\left(\mathbf{A}+\mathbf{V}_{R}\right) f=-f^{\prime \prime}(x)+f(x)+\left[b_{21} f(0)+b_{22}(f, q)\right] q(x)+\left[b_{11} f(0)+b_{12}(f, q)-f_{s}^{\prime}\right] \delta(x)$.

The $L_{2}(\mathbb{R})$-part of $\mathbf{A}+\mathbf{V}_{R}$ is a self-adjoint operator $\widetilde{A}$ in $L_{2}(\mathbb{R})$ determined by the rule:

$$
\widetilde{A} f=-f^{\prime \prime}(x)+f(x)+\left(b_{21} f(0)+b_{22}(f, q)\right) q(x),
$$

where $f \in \mathcal{D}(\widetilde{A})=\left\{f \in W_{2}^{2}(\mathbb{R})+N \mid b_{11} f(0)+b_{12}(f, q)=f_{s}^{\prime}\right\}$ and $\widetilde{A}$ can be interpreted as a model of the Schrödinger operator with non-local point interactions [3, 4].

\section{Acknowledgments}

The authors thank the referee for remarks which led to improvements in the paper. In particular the initially imposed assumption that the perturbation is of finite rank was removed. The second (S.K.) and third (L.N.) authors are grateful to DFG for the support of the projects 436 UKR 113/88/0-1 and 436 UKR 113/79, respectively. They also thank to scientific program of NAS of Ukraine project $0107 \mathrm{U} 002333$ for the support.

\section{References}

[1] S. Albeverio, F. Gesztesy, R. Høegh-Krohn and H. Holden, Solvable Models in Quantum Mechanics, Springer-Verlag, Berlin/New York, 1988; $2^{\text {nd }}$ ed. (with an appendix by P. EXNER), AMS Chelsea Publishing, Providence, RI, 2005.

[2 2 S. Albeverio and P. KuRASOv, Singular perturbations of differential operators. Solvable Schrödinger type operators, London Math. Soc. Lecture Note Ser. 271, Cambridge Univ. Press, Cambridge, 2000. 
[ 3 ] S. AlbeVerio and L. P. NizhniK, A Schrödinger operator with point interactions on Sobolev spaces, Lett. Math. Phys. 70 (2004), 185-199.

[4] S. Albeverio and L. P. NizhniK, Schrödinger operators with nonlocal point interactions, J. Math. Anal. Appl. 332 (2007) 884-895.

[ 5 ] T. ANDO and K. NiSHIO, Positive selfadjoint extensions of positive symmetric operators, Tohoku Math. J. 22 (1970), 65-75.

[ 6 ] YU. M. ARLinSKiI and E. R. TSEKANOVSKII, Some remarks of singular perturbations of self-adjoint operators, Methods Funct. Anal. Topology, 9, No. 4 (2003), 287-308.

[ 7 ] YU. M. BereZAns KiI, Expansions in Eigenfunctions of Self-Adjoint Operators, Transl. Amer. Math. Soc. 17, Providence, Rhode Island, 1968.

[ 8 ] M. S. BIRMAN, On the self-adjoint extensions of positive definite operators, Mat. Sbornik, 38 (1956), 431-450 (Russian).

[ 9 ] E. A. Coddington, Self-adjoint subspace extensions of nondensely defined symmetric operators, Advances in Math. 14, No. 3 (1974), 309-332.

[10] V. DeRKaCh and S. Hassi, H. DE SNoo, Singular perturbations of self-adjoint operators, Math. Physics, Analysis and Geometry 6 (2003), 349-384.

[11] V. A. Derkach and M. M. Malamud, Characteristic functions of almost solwable extensions of Hermitian operators, Ukrainian Math. J. 44 (1992), 379-401.

[12] E. Fermi, Sul moto dei neutroni nelle sostanze idrogenate, Ricerca Scientifica, 7 (1936), 13-52. (English translation in E. Fermi, Collected papers, Vol. I, Univ. of Chicago Press, Chicago, 1962, pp. 980-1016.)

[13] M. L. GorbachuK and V. I. GorbaChuk, Boundary-Value Problems for Operator-Differential Equations, Kluwer, Dordrecht, 1991

[14] P. R. Halmos, Finite-Dimensional Vector Spaces, D. van Nostrand Company, Princeton, 1961.

[15] S. HASSI and S. KUZHEL, On symmetries in the theory of singular perturbations, Preprint University of Vaasa, 2006 (http://www.uwasa.fi/julkaisu/).

[16] T. Kato Perturbation Theory of Linear Operators, Springer, Berlin, New-York, 1980.

[17] A. KisELeV and B. SimON, Rank one perturbations with infinitesimal coupling, J. Funct. Anal. 130 (1995), 345-356.

[18] M. KRASNOSEL'SKII, On self-adjoint extensions of Hermitian operators, Ukr. Mat. Zh. 1 (1949), 21-28.

[19] A. N. KochubeI, On extensions of nondensely defined symmetric operator, Sib. Math. J. 18, No. 2 (1977), 314-320.

[20] M. G. KREIN, The theory of self-adjoint extensions of semibounded Hermitian transformations and its applications I, Mat. Sbornik 20 (1947), 431-495 (Russian).

[21] P. KURASOV and S. T. KURODA, Krein's resolvent formula and perturbations theory, J. Oper. Theory 51, No. 2 (2004), 321-334.

[22] S. T. Kuroda and H. NAGAtani, $\mathcal{H}_{2}$-construction of general type and its application to point interactions, J. Evol. Equ. 1 (2001), 421-440.

[23] A. KUZHEL and S. KUZHEL, Regular Extensions of Hermitian Operators, VSP, Utrecht, 1998.

[24] S. KuZHel and L. NizhniK, Finite rank self-adjoint perturbations, Meth. Func. Anal. Topology 12 (2006), no. 3, 223-241.

[25] L. P. NIZHNIK, On rank one singular perturbations of self-adjoint operators, Meth. Func. Anal. Topology 7 , No. 3 (2001), 54-66.

[26] L. P. NiZHNiK, One-dimensional Schrödinger operators with point interactions in the Sobolev spaces, Funct. anal. appl. 40, No. 2 (2006), 74-79.

[27] A. Posilicano, Self-adjoint extensions by additive perturbations, Ann. Scuola Norm. Sup. Pisa Cl. Sci. Vol. II (5) (2003), 1-20. 
Present Addresses:

SERGio AlbeVerio

InSTITUT FÜR ANGEWANDTE MATHEMATIK,

UNIVERSITÄT BONN.

WEGELERSTR. 6, D-53115, BONN, GERMANY.

SFB 611, BONN, BIBOS, BIELEFELD-BONN.

CERFIM, LOCARNO AND USI, SWITZERLAND.

e-mail: albeverio@uni-bonn.de

SERGEI KUZHEL

InSTITUTE OF MATHEMATICS OF THE NATIONAL ACADEMY OF SCIENCES OF UKRAINE,

TERESHCHENKOVSKAYA 3, 01601 KIEV, UKRAINE.

e-mail: kuzhel@imath.kiev.ua

LEONID P. NIZHNIK

InSTITUTE OF MATHEMATICS OF THE NATIONAL ACADEMY OF SCIENCES OF UKRAINE,

TERESHCHENKOVSKAYA 3, 01601 KIEV, UKRAINE.

e-mail: nizhnik@imath.kiev.ua 